\title{
Die spätwürmeiszeitlichen Stadien auf der schweizerischen Alpennordseite
}

\author{
Von René Hantke, Zürich
}

Mit 3 Abbildungen

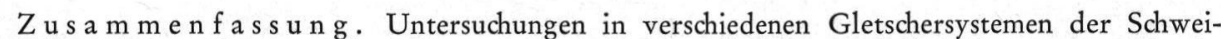
zerischen Alpennordseite ergaben eine gute Korrelation zwischen entsprechenden spätwürmeiszeitzeitlichen Rückzugsstadien. Die ausgeprägten Moränen des Zürich-Stadiums, rund $15 \mathrm{~km}$ hinter dem Maximalstadium des würmeiszeitlichen Linthgletschers, dämmen, wie die Stirnmoränen des entsprechenden Stadiums in den anderen Systemen, Seebecken ab, die während älteren Eisvorstößen ausgekolkt wurden. Das nächste Rückzugsstadium, dasjenige von Hurden, konnte auch andern Systemen zuerkannt werden. Ebenso zeichnen sich die zuerst in den subaquatischen Moränen des Vierwaldstättersees erkannten Rückzugsstadien durch Moränen, Schmelzwasserrinnen und Schotterfluren auch in den übrigen Gletschersystemen ab. Während einer länger andauernden wärmeren Periode begannen die Gletscher abzuschmelzen und zogen sich tief in die Alpentäler zurück. Längs Kluftflächen brachen dabei die ihres Gegendruckes durch das Eis verlustig gegangenen Talflanken als Bergsturzmassen nieder.
\end{abstract}

Eine deutliche Klimaverschlechterung ließ die Gletscher erneut vorstoßen, den Vorderrheingletscher gar um mehr als $40 \mathrm{~km}$ über die Bergsturzmassen von Flims bis Chur, wo er durch den ebenfalls vorrückenden Plessurgletscher gebremst wurde.

Künftige palynologische und tephrochronologische Untersuchungen in geschützten seitlichen Zungenbecken werden erlauben, die einzelnen Stadien auch chronologisch einzustufen.

$\mathrm{A} \mathrm{b} \mathrm{s} \mathrm{r}$ a ct. Investigations on late Würmian readvance stages of the glaciers on the northern slope of the Swiss Alps have shown that correlations can be made between the different glacier systems.

The well developed end moraines of the Zurich stage, 15 to $20 \mathrm{~km}$ behind the outermost Würmian moraines, are damming lake basins that had already formed during older ice advances. The next readvance, the Hurden stage, has also been recognized in all glacier systems. End moraines of still younger readvances, discovered first as subaquatic ridges in Lake Lucerne, likewise exist in the other system.

During a prolonged warmer period the glaciers retreated far upwards into the Alpine valleys. Due to the removal of the ice, rock masses of many valley slopes became unstable and broke down forming landslides.

A subsequent cooler time caused the glaciers to advance again. So the Vorder Rhine glacier overrode the landslide masses of Flims and advanced more than $40 \mathrm{~km}$ beyond Chur, where it was stopped by the Plessur glacier advancing from the SE side.

Palynological and tephrochronological investigations now in progress will permit to define the place of all the different stages in the absolute time scale.

\section{Einleitung}

In neuerer Zeit wurden im schweizerischen Alpenvorland mehrfach Versuche unternommen, die Endmoränenlagen in den einzelnen eiszeitlichen Gletschersystemen zeitlich $\mathrm{zu}$ korrelieren. Dabei ergaben sich schon hinsichtlich der Abfolge der gut ausgebildeten hochwürmeiszeitlichen Stadien bedeutende Differenzen. Diese beruhen vor allem auf der noch immer unvollständigen Kenntnis der einzelnen Ablagerungen in den verschiedenen Systemen und deren genetischer Verknüpfung mit einem reichen glazifluviatilen Formenschatz im Vorland und längs des Gletscherrandes.

Uber würmeiszeitliche Vorstoßhalte wurden bisher erst wenige Versuche gewagt (Hantke 1959, 1962, KläY 1969), die es noch weiter zu festigen gilt. Eisvorstöße sind weitgehend mitverantwortlich für die Ausgestaltung tieferer Wannen. Dadurch wurden auch die Rückzugshalte bereits deutlich vorgezeichnet, die sich heute in Talkesseln oder gar noch in persistierenden Seewannen zu erkennen geben. 
Ebenso gingen die Meinungen über die Korrelation und die zeitliche Einstufung der späteiszeitlichen Eisrandlagen ziemlich auseinander. In den letzten Jahrzehnten wurde auch den spät- und postglazialen Moränenständen und damit den Klimaschwankungen der ausgehenden Würmeiszeit und des Postglazials vermehrte Aufmerksamkeit geschenkt (Beck 1925\%, 1934, 1955, Salathé 1961, Zoller 1960, 1966, Hantke 1958, 1967, 1968, 1969, 1970), so daß diese nunmehr weitgehend geklärt sind. Eine chronologische Einstufung mit Hilfe pollenanalytisch untersuchter Profile in Zungenbecken ist im Gange.

\section{Das Stein am Rhein/Zürich-Stadium}

Unter den Moränenstadien der ausgehenden Hochwürmeiszeit zeichnet sich besonders das durch mehrere eng aufeinander folgende Staffeln charakterisierte Zürich- oder SeenStadium aus. Die einzelnen Moränenzüge lassen sich im Gelände ohne Schwierigkeit von einem Gletscherlappen zum nächsten und über Transfluenzsättel und an gemeinsamen Schotterfluren gar von einem Gletschersystem ins benachbarte verfolgen, etwa vom Saanengletscher hinüber zum Aare- und weiter über den Reuß- und den Linth- bis zum Rheingletscher, so daß an ihrer Gleichzeitigkeit nicht zu zweifeln ist (Abb. 1). Nur beim Rhonegletscher, besonders beim Solothurner Arm, treten die einzelnen Eisrandlagen dieses Stadiums etwas weniger markant in Erscheinung. Dies dürfte darauf zurückzuführen sein, daß der verantwortliche Klimarückschlag nur kurzfristig einwirken konnte und damit die Schuttmenge zu gering war, als daß sich die einzelnen Staffeln bei den gewaltigen Eismassen, die vom Genfersee-Becken über die Wasserscheiden des Jorat und von La Sarraz ins westliche Mittelland überflossen, manifestieren konnten. Zudem reichten weite Gebiete der Gletscheroberfläche bis in die Firnregion.

\section{Das Konstanz/Hurden-Stadium}

Auf das Seen abdämmende Zürich-Stadium folgen zunächst einige Rückzugsstaffeln, die sich durch drumlinartige Seitenmoränenzüge zu erkennen geben. Erst die zeitlichen Aquivalente des Konstanz/Hurden-Stadiums lassen sich in den einzelnen Gletschersystemen wieder mit einiger Sicherheit korrelieren (Abb. 1).

Damals endete der A a reglets che r bei Jaberg zwischen Bern und Thun. Zwischen Spiez und Thun vermochte der $\mathrm{K}$ anderglet s cher nochmals bis zum Aaregletscher vorzustoßen, wobei der gemeinsame Moränenwall bei Zwieselberg drumlinartig überprägt wurde. Am Ausgang des Simmentales stand der Diemtig/Simmen- noch mit dem Kandergletscher in schmaler Verbindung. Der $\mathrm{S}$ a a nenglets cher reichte bis über Enney S von Greyerz, wo mächtige Stauschotter, Wallmoränenreste und Rundhöcker ein Zungenbecken umsäumen.

Beim Hauptarm des R e u ß g l e t s c he r s zeichnet sich dieses Stadium bei GisikonHonau, beim Zugersee-Lappen bei Hünenberg-Baar und bei Rotkreuz ab.

Im $\mathrm{R}$ heins y s te m liegen die entsprechenden Stirnmoränen im Bodensee-Lappen in Konstanz, im Thur-Lappen bei Bürglen-Kradolf.

Der Thurgletscher reichte damals, dank eines Staues durch den Rheingletscher und einer geringfügigen Transfluenz von Rheineis, noch bis Nesslau (Hantke 1967).

Der Urnäschgletscher endete oberhalb des Dorfes Urnäsch, der Sitterglet scher unterhalb Appenzell.

Beim Rhonegletscher dürfte dieses Stadium den Eisrandlagen von YvoireNyon im Genfersee-Arm, von Mathod-Yverdon in der Zihl-Neuenburger-Talung und von Fétigny SW von Payerne im Broyetal entsprechen.

*) bedeutet geologische Karten, die im Literaturverzeichnis gesondert aufgeführt sind. 


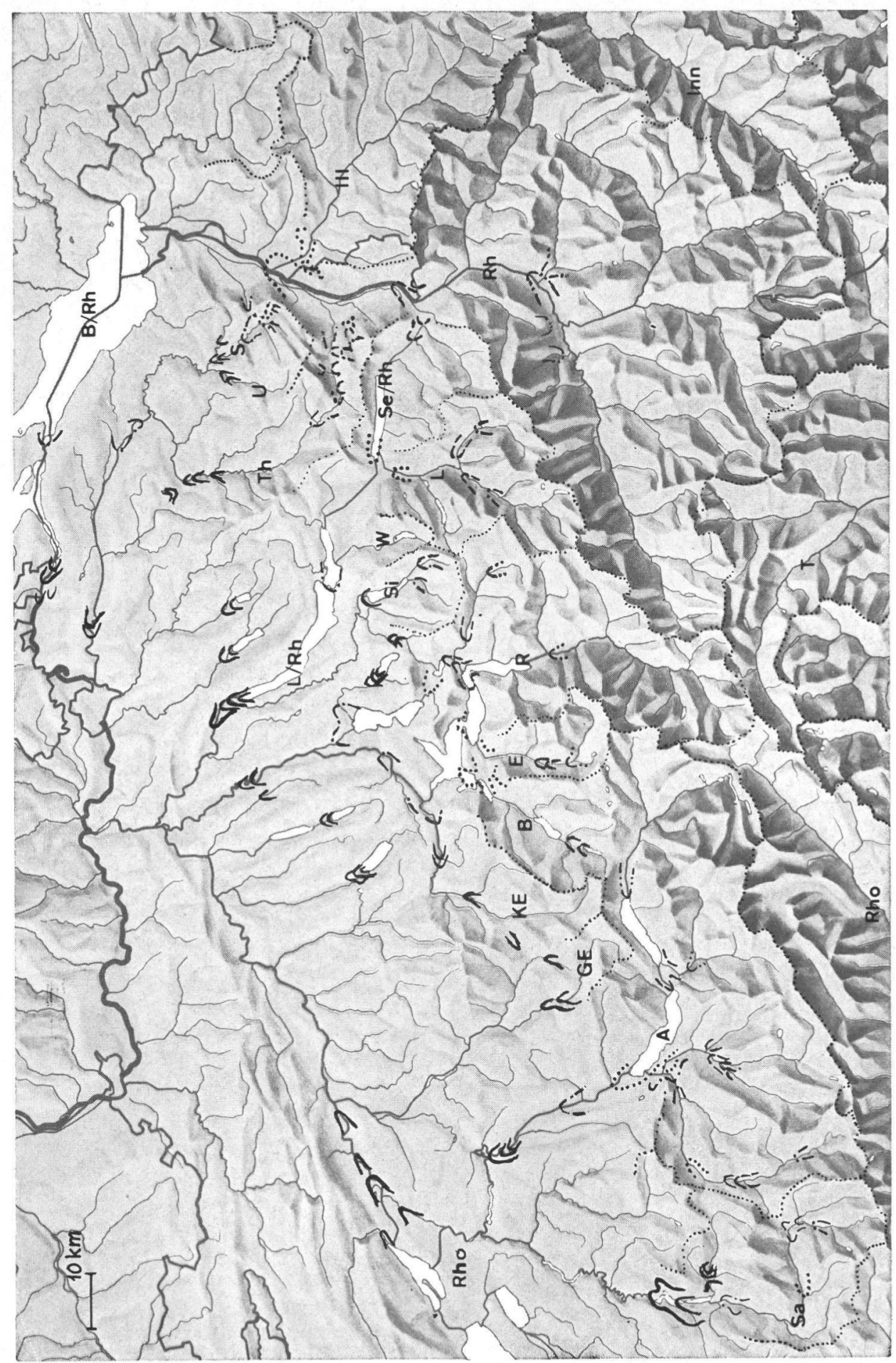

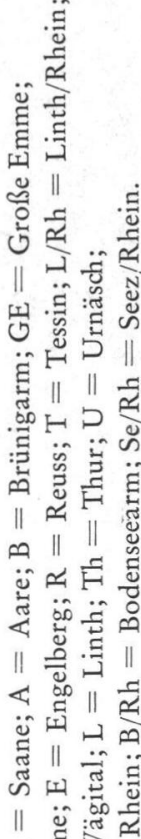

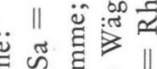

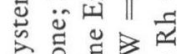
का .

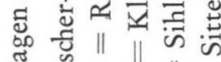

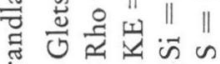
离

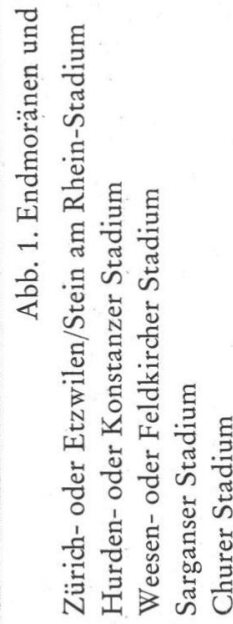
rics 


\section{Die unterseeischen Moränen im Vierwaldstättersee und ihre zeitlichen Äquivalente in anderen Gletschersystemen}

Über die weiter alpeneinwärts gelegenen Moränenstände, PENCKs Bühlstadium, herrschte - trotz ihrem frühen Erkennen in einzelnen Systemen - über deren Existenz in andern und erst recht über deren Parallelisation noch reichlich Unsicherheit. In den Haupttälern sind sie zum Teil später von holozänen Sedimenten völlig eingedeckt worden, oder sie reichen - wie im Vierwaldstättersee - nicht über den Wasserspiegel empor.

Im Vierwaldstätte rsee erkannte bereits Alb. Heim (1894, S. 180) in den beiden durch Lotungen festgestellten wallartigen Erhebungen bei Vitznau und $\mathrm{E}$ von Gersau unterseeische Endmoränen (Abb. 2). Der äußere Wall quert den See N der beiden Nasen und erhebt sich bis $27 \mathrm{~m}$ unter den Wasserspiegel aus einem $214 \mathrm{~m}$ tiefen Zungenbecken; der internere steigt aus dem $125 \mathrm{~m}$ tiefen Seebecken W von Brunnen beim Chindli bis auf $50 \mathrm{~m}$ unter den Wasserspiegel empor. Die beiden könnten zunächst einem einzigen Stadium angehören, da der Engelberger Gletscher neben dem Hauptarm von StansStansstad einen seitlichen Lappen über Buochs gegen NE und gegen E vorschob. Dabei wäre es $\mathrm{N}$ der Nasen zur Bildung der Vitznauer Endmoräne gekommen, während der gegen $\mathrm{E}$ vorgestoßene Arm den Reußgletscher am weiteren Vorrücken gegen W gehindert hätte. Die Gersauer Moräne wäre dann als unterseeischer Stauwall zwischen Engelberger Gletscher und Reußgletscher zu deuten.

In der Talung Brunnen-Lauerzersee-Zugersee, durch die neben dem Muotagletscher auch ein bedeutender Anteil von Reuß-Eis abfloß, läßt sich im Lauerzersee ein Zungenbecken erkennen, das bei Goldau durch eine Molasseschwelle und durch die Roßberg-Bergstürze noch akzentuiert wird. S von Steinerberg wird dieses Stadium durch zahlreiche Kristallin-Erratiker und SE von Lauerz durch seitliche Wallmoränenreste dokumentiert.

$\mathrm{Da}$ sich nun das Gersauer Stadium auch in der Talung Brunnen-Lauerzersee in den Seitenmoränen am Ausgang des Muotatales, in der Endmoräne von Ingenbohl, sowie in den Seitenmoränen am SE-Fuß des Urmiberges klar zu erkennen gibt, ist an der Existenz zweier zeitlich getrennter Stadien im Vierwaldstättersee nicht zu zweifeln. Der geradlinige Verlauf im See ist möglicherweise auf die Stauwirkung einer im Becken von Gersau gelegenen Toteismasse zurückzuführen (Hantke 1958, S. 126).

Am Ausgang des Muotatales stellen sich unter der markanten Reuß-Seitenmoräne mächtige Talrandschotter ein. Während sie Du PAsquier (1891, S. 107) noch als interglazial betrachtet hatte, erkannte bereits Buxtorf (1916), daß in diesen spätglaziale, vom Gletscher nur geringfügig überfahrene Eisrandschotter vorliegen.

Die Seitenmoränen des Gersau/Ibach-Ingenbohl-Stadiums zeichnen sich besonders im Ingenbohler Wald durch einen steilen Frontanstieg bis auf $670 \mathrm{~m}$ aus. Stauschotter am Ausgang des Isentales bei Birchi in $720 \mathrm{~m}$ und an der Mündung des Schächentales in $780 \mathrm{~m}$ belegen ein Vorrücken des Isental- und des Schächengletschers bis ins Haupttal. Bei beiden erfolgte über einen Querschnittsbereich noch ein Zufluß zum Reußgletscher. Nur dank derartiger Zuflüsse vermochte dieser überhaupt nochmals bis gegen Gersau und Ibach vorzurücken. Zudem dürfte im untersten Reußtal und im Urnersee-Becken noch ein gewisser Eisvorrat aus dem Vitznau/Goldau-Stadium gelegen haben. Dies war nur möglich, wenn die dazwischen gelegene Schwankung unbedeutend, kurzfristig und kühl gewesen war.

In gleicher Weise zeichnen sich die beiden Vierwaldstättersee-Stadien auch in der westlichen Zentralschweiz ab. Der über den Brünig geflossene Arm des A a reglets che rs, der im Vitznau/Goldau-Stadium noch einen Eiszuschuß aus den Melchtälern erhielt, stieß einerseits bis über Stansstad in den Vierwaldstättersee vor, andererseits drang ein Lappen zwischen Muoterschwanderberg und Stanserhorn bis Allweg W von Stans vor, wo er von einem Lappen des Engelberger Gletschers, der sich von Stans gegen $\mathrm{W}$ wandte, gestaut wurde (Abb. 2). 


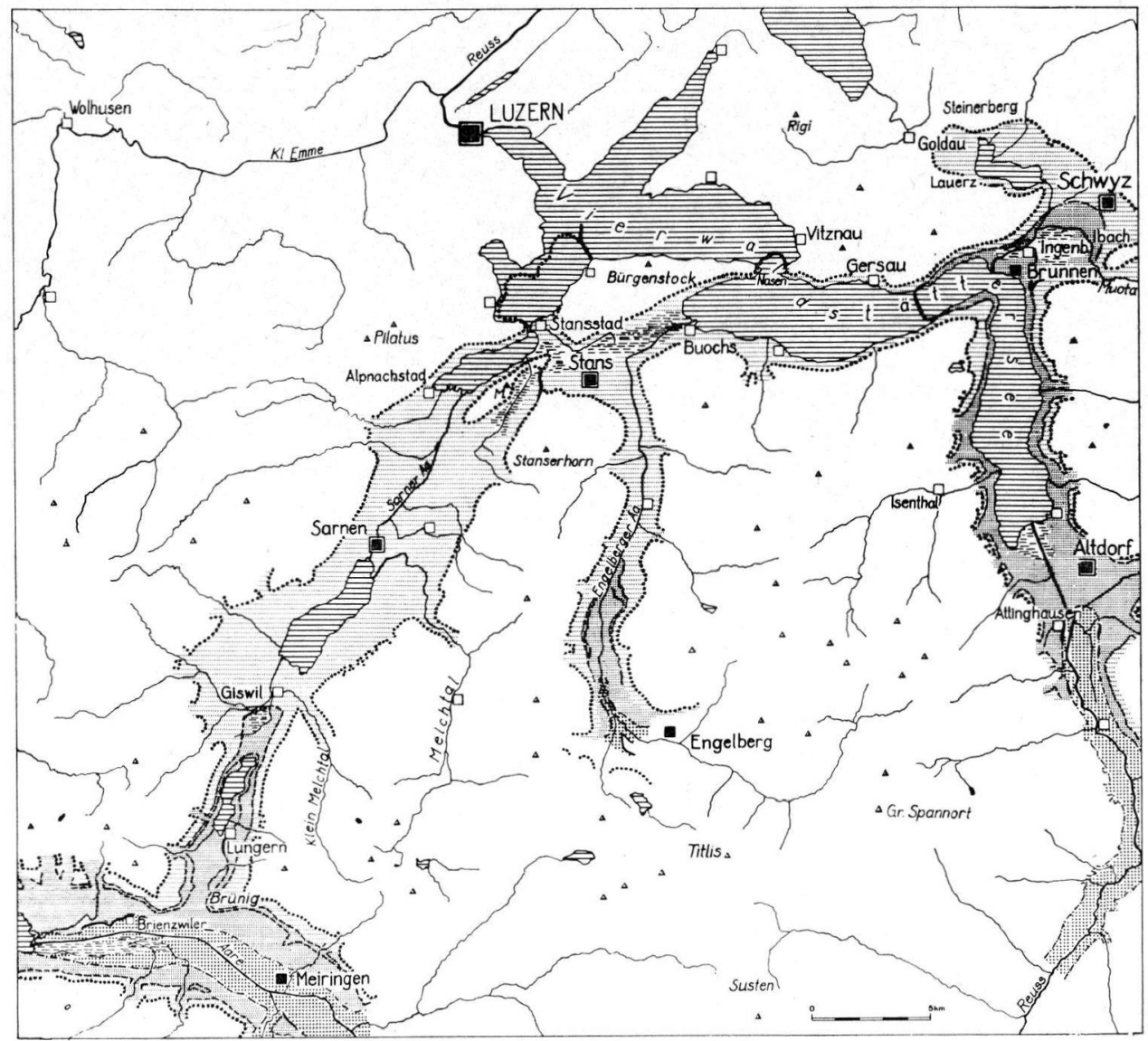

Abb. 2. Die spätwürmeiszeitlichen Stadien im Reuss-System und die Transfluenz des Aaregletschers.

Spätwürmeiszeitliche Moränen und Eisrandlagen

V Vitznau/Goldau- = Weesen/Feldkirch-Stadium

i) Gersau/Seewen - Ingenbohl- = Sarganser Stadium

-1 Attinghausen- $=$ Churer-Stadium.

Noch im Gersauer Stadium vermochte der Brünigarm des Aaregletschers bis Giswil vorzurücken, wo er das von Rundhöckern und Wallmoränenresten umsäumte Zungenbecken des Aariedes ausfüllte.

Während der Brünigarm bis Stansstad und Allweg vorstieß, rückte der A a r e g l e t s c h e r selbst, von kräftigen Zuflüssen aus den Lütschinentälern unterstützt, nochmals bis über Thun vor und schüttete $\mathrm{W}$ des Thunersees die linksseitige Ufermoräne von Strättligen, die ein Gletscherende unterhalb von Thun annehmen läßt, auf. Auch der K a n $\mathrm{d}$ e r g l e t s c h e r rückte damals bis zum Aaregletscher vor, wurde jedoch von diesem ganz an die NE-Flanke des Niesen gedrückt (BECK \& GERber 1925\%). Einzig die Zunge konnte sich NE von Wimmis freier entfalten. Der Sim menglet s cher vermochte nochmals bis unterhalb Boltigen vorzustoßen, während der Diemtigglets cher gar den Talausgang bei Oey erreichte. Der $\mathrm{S}$ a a n e nglets cher endete damals bei Les Moulins unterhalb Château d'Oex (Abb. 1). 
Das Ende des R ho n e g le t s c her s dürfte sich in den glazifluviatilen Ablagerungen von Noville-Chessex zu erkennen geben, während die Wallmoränenreste von einem damals noch bis ins Rhonetal vorgestoßenen Lokalgletscher stammen (M. BURRI 1961).

Altersgleiche Moränenstadien, die den beiden Stadien von Vitznau/Goldau und von Gersau/Ibach-Ingenbohl entsprechen, zeichnen sich auch im Bereich des R heingletschers ab (Arn. Heim 1907*; Hantke 1968, 1970). In der Walensee-Talung stellte die frühere Insel Hutteboschen bei Weesen (SCHeUchzer 1712\%), die später angelandet und bei der Linthkorrektion eingeebnet wurde, einen Rest einer Stirnmoräne dar (Abb. 1). Der Walensee ist als zugehöriges Zungenbecken zu deuten; die Moränenwälle von Betlis und von Buechen WSW von Murg entsprechen Seitenmoränenresten beidseits des Sees. Das spätere Gersau/Ibach-Ingenbohl-Stadium manifestiert sich im Seeztal in Moränenwällen, Schmelzwasserrinnen und Schotterfluren zwischen Flums und Sargans, während sich das ältere Stadium dort in Stauschutt-Terrassen und höher gelegenen Rundhöcker-Arealen an den Talausgängen abzeichnet.

Im Rheintal endete der Rheingletscher zunächst bei Feldkirch, wo ein Seitenlappen einem Stirnlappen des Illgletschers gegenüberstand. Das jüngere Stadium gibt sich im Becken von Balzers-Azmoos und in der Talenge zwischen Schollberg und Fläscherberg zu erkennen (HANTKE 1968, 1970).

Im Gebiet des Linthgletschers entsprechen die Eisrandlagen von NäfelsMollis derjenigen des Vitznauer Stadiums, jene von Nidfurn - wo sich im Liegenden wiederum eisrandnahe Schotter einstellen - und von Leuggelbach im Großtal, von Wart und Engi im Kleintal, demjenigen von Gersau/Ibach-Ingenbohl (Abb. 1).

Der A a reglets cher endete in den beiden, dem Stadium von Gersau/IbachIngenbohl entsprechenden Eisrandlagen zunächst am Ostende des Thunersees, später im Raume von Interlaken, was durch tiefgelegene Rundhöcker, 2 Systeme von tiefen Wallmoränen und Schotterterrassen S von Bönigen, bei Gsteigwiler, bei Wilderswil und S von Interlaken bekundet wird (GüNZLER-SEIFFERT 1933*). Der K a n de rglets che r vermochte damals eben noch den Engstligengletscher aufzunehmen und schob seine Zunge bis unterhalb Frutigen vor. Beim Si m m e n l e t s cher lagen die entsprechenden Zungenenden unterhalb $\mathrm{Z}$ weisimmen, beim $\mathrm{S}$ a a n e $\mathrm{n} \mathrm{l}$ e $\mathrm{t} \mathrm{s} \mathrm{ch}$ e r unterhalb Saanen.

Der Rhonegletscher stieß während dieses Stadiums nochmals bis Aigle, bzw. Collombey-Ollon vor. Beidseits des Rhonetales absteigende Seitenmoränen bekunden dort Eisrandlagen.

\section{Das Churer Stadium}

Ein noch späteres, wiederum durch Schotterfluren gekennzeichnetes Stadium wurde von R. Staub (1938) in der Gegend von Chur postuliert und mit v. Klebelsbergs (1927) SchlernStadium in Verbindung gebracht. Dieses wird jedoch neuerdings von einigen Autoren wieder fallen gelassen, da der Schlerngletscher in Südtirol bei einer Schneegrenzen-Depression von $900 \mathrm{~m}$ noch mit dem Eisackgletscher verbunden war, was nach MAYR \& HeUBerger (1968) und Heuberger (1968) eher einer „Bühl-Situation“" entspricht.

Nach STAub erfolgte der Churer Vorstoß auf einen kräftigen Eisschwund, bei dem der Vorderrhein-Gletscher sich bis hinter Ilanz zurückgezogen und in mehrere Stammgletscher aufgelöst hätte, also auf ein deutliches Interstadial. Dabei verloren die Bergflanken, die durch Talklüftung in ihrem Verbande gelockert waren, ihren Gegendruck durch das Gletschereis und brachen als Bergstürze nieder. Im Churer Stadium stieß dann der Vorder rhein-G let scher, von Reichenau an unterstützt durch den durchs Domleschg vorrückenden $\mathrm{H}$ inter rhein-G letscher, erneut bis Chur vor (Abb. 3). Dabei wurden die Bergsturzmassen von Flims und von Reichenau-Chur vom Eis über- 
fahren, was durch aufliegende Moräne mit Erratikern bekundet wird. Im bewegungsarmen Stirnbereich kam es, infolge der Stauwirkung durch den ebenfalls bis Chur vorrückenden Plessurgletscher, zur Bildung der Tomas von Ems, Felsberg und Chur.

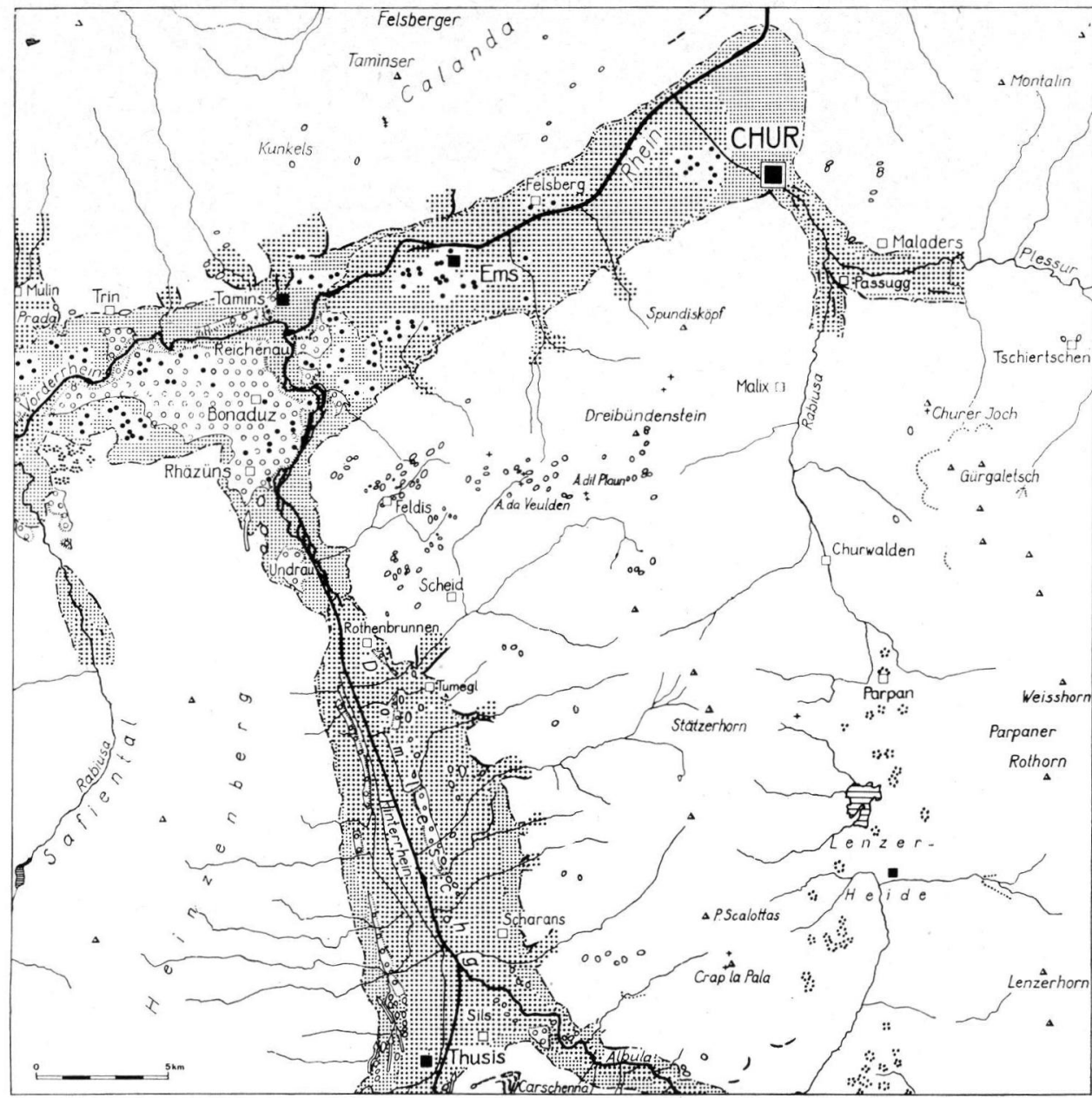

Abb. 3. Die spätwürmeiszeitlichen Stadien im Rhein-System zwischen Thusis und Chur.

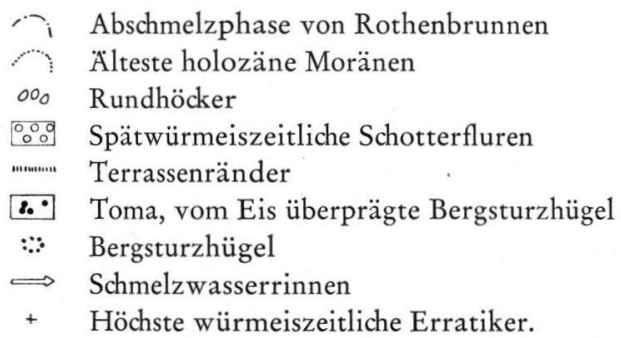

Stirnmoränenreste W und NE von Tamins weisen darauf hin, daß auch aus der Ringelspitzkette nochmals steile Gletscher bis in die Zungenbecken von Prada Mulin und Girsch vorstießen, wo sie durch den Rheingletscher gestaut wurden. Ebenso läßt sich $\mathrm{N}$ von Ems 
die linke Seitenmoräne eines Hängegletschers vom Taminser Calanda bis auf $700 \mathrm{~m}$ herab verfolgen. Im Domleschg werden die von der Stätzerhornkette absteigenden Lokalgletscher an den Talausgängen zwischen Scharans und Tumegl/Tomils durch Seitenmoränen dokumentiert, die vom Hinterrhein-Gletscher in dessen Stromrichtung abgewinkelt worden waren (Abb. 3).

Bei einer ersten Abschmelzphase schmolz dann der Rheingletscher an den dünnsten Stellen - über den Bergsturzmassen von Reichenau, bei Rhäzüns und über dem Talausgang des Safientales - durch, so daß Toteis abgetrennt wurde. Durch die Bergsturz- und Toteismassen wurden dort Eisrandschotter aufgestaut bis die Erosionsbasis durch das Abschmelzen des Churer Toteises tiefer gelegt worden war; dann wurden die Schotterfluren kräftig zerschnitten.

Im Linthsystem mochten die Talgletscher damals erneut bis Luchsingen im Linthtal und bis Matt im Sernftal gereicht haben.

Im R e us t a 1 entspricht dieser Vorstoß dem mit W. BRücKner festgestellten Gletscherende von Attinghausen, das durch Stauterrassen und tiefliegende Seitenmoränen beidseits der Reuß dokumentiert wird. Im Tal der Engelberger Aa bekunden Seitenmoränenreste in $800 \mathrm{~m} \mathrm{NW}$ Engelberg diesen späten Vorstoß.

Im Berner Oberland vermochten sich damals die beiden großen Eislieferanten des Aaregletschers, der Hauptarm aus dem Haslital und jener aus den Lütschinentälern, nicht mehr zu vereinigen. Der A a regletscher, dokumentiert durch absteigende Moränen S des Brünigpasses (Arbenz 1911\%), bei Brienzwiler und auf dem Ballenberg (Michel 1921*) dürfte noch bis ans Ostufer des Brienzersees gereicht haben, während der

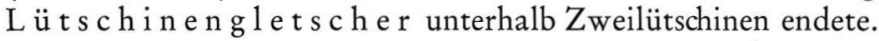

Der Rhonegletscher mag nochmals bis zur Talenge von St. Maurice gereicht haben.

\section{Chronologische Einstufung der spätglazialen Eisstände}

Auf eine länger anhaltende Erwärmungsphase, in der die Eismassen des Schweizerischen Mittellandes bis in die Alpentäler hinein abzuschmelzen begannen (Prä-Bölling?), erfolgten erste Klima r ü ckschläge. Da die spätwürmeiszeitlichen Gletscherstände - besonders ausgeprägt etwa im Rheintal und bei den Seitengletschern - durch auffällige Vorstöße gekennzeichnet werden, bekunden sie eine nur kurzfristige Klimaverschlechterung mit verschiedenen Schüben. Auf diese ersten Klimarückschläge folgte zunächst eine längere Erwärmungszeit, welche die Gletscher zurückweichen und sich in einzelne Eisarme auflösen ließ (Bölling?). Dann erfolgte, wie in verschiedenen Gletschersystemen zu erkennen ist, ein $\mathrm{z}$ we it e r deut licher Klimar ü ckschlag, so daß die Gletscher über ausgedehnte Bergsturzareale wieder vorstießen (Ältere Dryaszeit?).

Einige seitlich der Hauptarme gelegene Zungenbecken blieben nun auch auf der schweizerischen Alpennordseite vor einer späteren Zuschüttung bewahrt. Dort ist das Abteufen von Bohrprofilen bis auf die Moräne möglich, welche eine zeitliche Einstufung pollenanalytisch, tephrochronologisch oder mittels Radiokarbon-Datierungen ermöglichen. Damit können die vorwiegend morphologisch gewonnenen Resultate paläobotanisch gestützt werden.

\section{Literaturverzeichnis}

BECK, P.: Das Quartär. In: Geologischer Führer der Schweiz, 1, 26-41, Basel 1934.

- : Regionale Grundlagen für die Gliederung des alpinen Quartärs. - Eclogae geol. Helv., 47/2 (1954), 369-373, Basel 1955.

Burri, M.: Les dépôts quaternaires de la vallée du Rhône entre St-Maurice et le Léman. - Bull. La Murithienne, 78, 36-59. 
Buxtorf, A.: Erläuterungen zur geologischen Karte der Rigihochfluhkette. - Geol. Karte Schweiz, 14, Bern 1916.

Du Pasquier, L.: Über die fluvioglazialen Ablagerungen der Nordschweiz. - Beitr. geol. Karte Schweiz, N. F., 1, Bern 1891.

HantKe, R.: Die Gletscherstände des Reuss- und Linthsystems zur ausgehenden Würmeiszeit. Eclogae geol. Helv., 51/1, 119-149, Basel 1958.

- : Zur Phasenfolge der Hochwürmeiszeit des Linth- und Reuss-Systems, verglichen mit derjenigen des Inn-Salzach-Systems sowie der nordischen Vereisung. - Vjschr. naturf. Ges. Zürich, 104/4, 390-402, Zürich 1959.

- : In: Suter, H./HantKe, R.: Geologie des Kantons Zürich. Zürich 1962.

- : Die würmeiszeitliche Vergletscherung im oberen Toggenburg (Kt. St. Gallen). - Vjschr. naturf. Ges. Zürich, 112/4, 223-242, Zürich 1967.

- : Die Diffluenz des würmeiszeitlichen Rheingletschers bei Sargans (Kanton St. Gallen) und die spätglazialen Gletscherstände in der Walensee-Talung und im Rheintal. - Eiszeitalter u. Gegenwart, 19, 219-226, "Öhringen/Württ. 1968.

- : Geschichte der Landschaft. In: Die Jüngere Steinzeit. Ur- und Frühgeschichtliche Archäologie der Schweiz, 2, 5-16, Zürich 1969.

- : Die Diffluenz des würmeiszeitlichen Rheingletschers bei Sargans und die spätglazialen Gletscherstände in der Walensee-Talung und im Rheintal. - Vjschr. naturf. Ges. Zürich, 115/1, 101-126, Zürich 1970.

Heim, Alb.: Ưber das absolute Alter der Eiszeit. - Vjschr. naturf. Ges. Zürich, 39/2, 180-186, Zürich 1894.

— : Geologie der Schweiz, 1, Leipzig 1919.

Heuberger, H.: Die Alpengletscher im Spät- und Postglazial. - Eiszeitalter u. Gegenwart, 19, 270-275, Öhringen/Württ. 1968.

JäckLI, H.: Exkursion Nr. 39, Teilstrecke I: Reichenau-Domleschg-Thusis-Via Mala-Zillis. Geol. Führer Schweiz, 8, 786-789, Basel 1967.

KLÄY, J.-R.: Quartärgeologische Untersuchungen in der Linthebene. Diss. ETH, Zürich, Uster 1969.

Klebelsberg, R. v.: Beiträge zur Geologie der Südtiroler Dolomiten. 1. Rückzugsstände der Eiszeitgletscher in den Dolomitentälern. - Z. deutsch. geol. Ges., 79 (1927), 280-354, Berlin 1928.

Mayr, R. \& Heuberger, H.: Type Areas of Late Glacial and Post-Glacial Deposits in Tyrol, Eastern Alps. - Proceed. VII Congress, 14, Internat. Union Quaternary Research Boulder Denver, Colorado, 1965, 143-165, Boulder 1968.

Nabholz, W. K.: Exkursion Nr. 38, Teilstrecke Chur-Reichenau. - Geol. Führer Schweiz, 8, 744-750, Basel 1967.

Pavoni, N.: Über die Entstehung der Kiesmassen im Bergsturzgebiet von Bonaduz-Reichenau (Graubünden). - Eclogae geol. Helv., 61/2, 494-550, Basel 1968.

Penck, A. \& Brǘckner, E.: Die Alpen im Eiszeitalter, 2, Leipzig 1909.

RemenYIK, T.: Geologische Untersuchung der Bergsturzlandschaft zwischen Chur und Rodels (Graubünden). - Eclogae geol. Helv., 52/1, 177-235, Basel 1959.

SALATHÉ, R.: Die stadiale Gliederung des Gletscherrückganges in den Schweizer Alpen und ihre morphologische Bedeutung. - Verh. naturf. Ges. Basel, 72/1, 157-231, Basel 1961.

Staub, R.: Altes und Neues vom Flimser Bergsturz. - Verh. schweiz. naturf. Ges. Chur, 60-85, Bern 1938.

- : Der Pass von Maloja. Seine Geschichte und Gestaltung. - Jber. naturf. Ges. Graubünden, 83, 3-84, Chur 1952.

StAUв, W.: Rückzugsstadien des würmeiszeitlichen Rhonegletschers im Gebiet zwischen Neuenburgersee und Genfersee. - Mitt. naturf. Ges. Bern, N. F., 5, 1-5, Bern 1948.

- : Die drei Hauptstadien des Rhonegletschers im schweizerischen Mittelland zur letzten Eiszeit. - Ber. Geobot. Forsch.-Inst. Rübel, (1949), 110-114, Zürich 1950.

ZimmermanN, H. W.: Die Eiszeit im westlichen zentralen Mittelland. - Mitt. naturf. Ges. Solothurn, 21, 3-143, Solothurn 1963.

Zoller, H.: Pollenanalytische Untersuchungen zur Vegetationsgeschichte der insubrischen Schweiz. - Denkschr. schweiz. naturf. Ges., 83/2, 45-156, Zürich 1960.

Zoller, H., Schindler, C. \& Röthlisberger, H.: Postglaziale Gletscherstände und Klimaschwankungen im Gotthardmassiv und Vorderrheingebiet. - Verh. naturf. Ges. Basel, 77/2, 97164, Basel 1966.

$$
\text { Geologische Karten }
$$

Arbenz, P.: Geologische Karte des Gebirges zwischen Engelberg und Meiringen, 1:50 000. Geol. Spez.-Karte, 55. Schweiz. Geol. Komm., Bern 1911 \%.

- : Geologische Karte der Uriotstockgruppe, 1:50 000. Geol. Spez.-Karte, 84, Ibid. 1918 *.

BECK, P.: Eine Karte der letzten Vergletscherung der Schweizeralpen. - Mitt. naturw. Ges. Thun, 1, Thun $1926 \%$. 
Beck, P. \& Gerber, Ed.: Geologische Karte Thun-Stockhorn, $1: 25000$. - Geol. Spez.-Karte, 96. Schweiz. Geol. Komm., Bern 1925 \%.

Buxtorf, A., Tobler, A., Niethammer, G., Baumberger, E., Arbenz, P. \& Staub, W.: Geologische Vierwaldstättersee-Karte, $1: 50000$. - Geol. Spez.-Karte, 66a, Ibid. $1916 \%$.

GünZler-SeIfFert, H.: Blatt 395: Lauterbrunnen. Geol. Atlas Schweiz, $1: 25$ 000, Ibid. 1933 *.

Heim, Arn. \& Oberholzer, J.: Geologische Karte der Gebirge am Walensee, $1: 25000$. Geol. Spez.-Karte, 44, Ibid. $1907 \%$.

Michel, F. L.: Geologische Karte und Profile des Brienzergrates, $1: 50000$. Geol. Spez.-Karte, 95, Ibid. $1921 \%$.

Oberholzer, J.: Geologische Karte des Kantons Glarus, $1: 50000$ et al. (1942\%). Geol. Spez.Karte, 117, Ibid. 1942\%.

Topographische Karten

Scheuchzer, J. J.: Nova Helvetia Tabula geographica ..., ca. 1 : 238 000, Zürich 1712\%.

Landeskarte der Schweiz $1: 25$ 000, Bern.

Manuskr. eingeg. 5. 8. 1970

Anschrift des Verf.: Prof. Dr. R. Hantke, Geol. Institut der Eidg. Technischen Hochschule Zürich, $\mathrm{CH} 8006$ Zürich, Sonneggstraße 5.

Erweiterte Fassung des Vortrages, gehalten anläßlich des VIII. INQUA-Kongresses in Paris 1969. 